\title{
IS PHENOMENAL CONSCIOUSNESS A COMPLEX STRUCTURE?
}

\author{
Chuck Stieg* \\ Department of Philosophy, University of Minnesota, Minneapolis, MN 55455, USA \\ Received September 21, 2009; accepted October 14, 2009
}

\begin{abstract}
Evolutionary explanations of psychological phenomena have become widespread. This paper examines a recent attempt by Nichols and Grantham (2000) to circumvent the problem of epiphenomenalism in establishing the selective status of consciousness. Nichols and Grantham (2000) argue that a case can be made for the view that consciousness is an adaptation based on its complexity. I set out this argument and argue that it fails to establish that phenomenal consciousness is a complex system. It is suggested that the goal of establishing consciousness as an adaptation may be better served by rejecting the distinction between access consciousness and phenomenal consciousness.
\end{abstract}

Key words: Consciousness; Complexity; Adaptation

\section{INTRODUCTION}

Evolutionary explanations of psychological phenomena have become fairly widespread, as have critiques of those same explanations (Barkow et.al 1992, Davies 1999, Fodor 2000, Godfrey-Smith 1996, Grantham and Nichols 1999, Okasha 2003, Shapiro 1999). The evolutionary origin of consciousness is one such phenomenon. The search for evolutionary explanations of consciousness is fueled by questions such as 'why are humans conscious?' and 'is consciousness an adaptation?' (see, for example, Polger and Flanagan 1999). To be an adaptation, consciousness must have some effects which have been selected for such that consciousness has evolved via natural selection as opposed to its presence being explained in other ways such as an exaptation, a spandrel, or by random drift and chance. It would help to make the case that consciousness is an adaptation if there was an obvious function that it served or effects which it had, however, the threat of epiphenomenalism makes this difficult.

In this paper, I will examine a recent attempt to circumvent the problem of establishing the selective status of consciousness based on postulating a function. Nichols and Grantham (2000) argue that a case can be made for the view that consciousness is an adaptation based on its complexity. Since the only scientifically respectable explanation for a complexly structured entity is that of evolution by natural selection, they conclude that consciousness is an adaptation. The examination of this argument will proceed as follows.
First, I will set out the problem of epiphenomenalism which motivates Nichols and Grantham's argument. Second, I will present their argument in detail and follow this up with a series of objections. The objections, roughly, can be categorized as falling under two general types. The first concerns the character of functional descriptions of cognitive processes and the assumption of modularity and the second concerns questions of consistency.

Questions regarding the need for adaptive explanations of consciousness are another possible route of criticism which one might explore, however, I will not pursue that here. Nichols and Grantham accept that evolutionary explanations of psychological phenomena are plausible and informative. I will grant that for the purpose of this paper, but I will argue that the evidence which they use is not sufficiently rigorous enough to support the premise that phenomenal consciousness is a complex system. Thus, Nichols and Grantham's argument fails to establish that consciousness is an adaptation. However, their goal might better be served by arguing against Ned Block's (1995) distinction, which they accept, between access consciousness and phenomenal consciousness.

\section{THE PROBLEM OF THE FUNCTION OF CONSCIOUSNESS}

Consider a common case of typing on a computer. When one does this, they have an experience of doing this; the subject is conscious of her fingers hitting the

\footnotetext{
*Correspondence to: Chuck Stieg, email: stie0076@umn.edu
} 
keys on the keyboard and of the words rolling out on the screen in front of her. She is aware of the hum of the computer and of one of the many outlet cords running from the computer brushing up against her foot. What's more, all of these various occurrences seem to be somehow bound together into one experience: her experience. This phenomenon seems to be both a persistent and critical aspect of what it is to be someone. At first blush we might call this consciousness, or at least, an aspect of consciousness. Humans, and arguably other animals, have this property. In Nagel's (1974) way of putting it, there is something it is like to be a creature with consciousness. They have a perspective or subjective experiences. This is certainly an incomplete picture of what many take to be consciousness, and it might even be controversial, but it will do for now. ${ }^{1}$

A question to pose at this point is one which asks about the origin of consciousness. Why are we conscious? Is it a product of our brains, and if so, is it an adaptation or might it simply be a by-product of the complex neural mechanisms which have evolved in the history of our species? If it is an adaptation, then consciousness will need to be shown to play, or have played, some role in contributing to the fitness of its bearer. If it is a byproduct, or a free-rider, then it need not make, or have made, a causal contribution to the fitness of its bearer since its existence will be due to the presence and causal role of some other trait to which it is related. Unfortunately, it is not immediately clear how to disentangle these different answers to the problem. For instance, it might seem obvious that consciousness plays some role, that being conscious provides an advantage over not being conscious. However, this may simply be illusory as it is possible that there could be creatures much like us, walking about, searching, muttering, etc., who lack what we might call consciousness. The possibility of "zombies" such as these is supposed to show that we should not take as given that our having consciousness plays some role in our lives. After all, if consciousness is not necessary to "get things done", then why should we think that our consciousness is? Natural selection would not waste its time on irrelevant solutions to problems with much simpler answers. So maybe consciousness is nothing more than an epiphenomenon.

Whatever the merits, or dismerits, of zombie-talk are, the threat of epiphenomenalism seems to undermine attempts to create stories designed to display a function of consciousness. But what about good, old-fashioned, empirical data? Neuropsychological data on dissociations suggests that there are many cases of correlations between a lack of consciousness and certain behavioral/cognitive deficits. For example, Young (1994) surveys a variety of examples including blindsight. In blindsight, a subject has had part or all of striate cortex removed in one hemisphere of their brain.
This causes a loss of vision for a part of their visual field, that is, the subjects report not being able to see anything in that area of their visual field. However, these subjects can still test well above chance on various tasks requiring them to respond to questions about stimuli in the damaged visual field, but they still perform poorly relative to their responses of stimuli in their undamaged visual field and relative to those who have not suffered damage to their brain. This is a case where there is a lack of phenomenal awareness, or consciousness, of what is in the damaged visual field, i.e. they are not aware of seeing anything there. Furthermore, their ability to respond to tests is less impressive than undamaged patients with full phenomenal consciousness of what they see. So consciousness might have some function related to this ability. Moreover, and more importantly, blindsight patients seem to lack the ability to initiate actions based on the information in their blind-field and can only use that information when prompted. This seems to suggest that an even deeper function of consciousness is to take in information, organize it in some particular way and to use it to initiate action in appropriate ways.

Though this might appear to be a promising strategy, there are potential problems again. Block (1995) argues that reasoning of the above sort is fallacious because it conflates two distinct notions of consciousness: access consciousness and phenomenal consciousness. The former is a state where its content, or what is represented by the state, can be used in reasoning or in rational control of action (Block, 1995, p. 229). Phenomenal consciousness ( $\mathrm{p}$-consciousness) is something like experience. P-conscious properties involve the experiential properties of perceptions, sensations and feelings, and we have p-conscious states when we see, touch, hear, etc. (Block, 1995, p. 230). According to Block, the reasoning in the above paragraph mistakes the function of access consciousness (a-consciousness) for the function of phenomenal consciousness. In such cases a-consciousness is also missing, and the function attributed to p-consciousness is more likely attributable to a-consciousness. So the neuropsychological data cannot be used to establish a function for p-consciousness. Block points out that there are many examples of this conflation in both the philosophical and psychological literature.

THE STRUCTURAL COMPLEXITY ARGUMENT Given Block's distinction it seems that there are now even more worries about whether phenomenal consciousness is an adaptation, what the function of consciousness is and how to determine its function. However, Nichols and Grantham (2000) offer an interesting and creative argument that phenomenal consciousness is an adaptation based on its complexity. They acknowledge the difficulties listed above but 
argue that if phenomenal consciousness can be shown to be a complex structure, then the only adequate explanation of its being such a complex structure is that it is an adaptation. In this section, I will lay out and explain their argument before turning to a critique of it in the following section.

According to Nichols and Grantham, evolutionary biologists often infer that a biological structure, if sufficiently complex, is an adaptation even if it is unknown what the function of the structure is. They provide an example of such an inference in the case of the lateral line system of fish and point out that even though it was unknown what the function of the system was, the structure of the system is sufficiently complex and constant throughout certain fish taxa to warrant the conclusion that it is an adaptation (Nichols \& Grantham, 2000, pp. 656-659).

The lateral line system in fish is made up of many interconnected parts. The lateral line canal extends down the length of the side of the fish and in the canal are many small organs which are each connected to a lateral line nerve (Nichols \& Grantham, 2000, p. 657). The spatial relations of the parts are maintained in conspecific organisms such that the canals are at the surface of the fish, and the organs extend into the canal and connect to a nerve (Nichols \& Grantham, 2000, p. $657)$. The structure of the system appears to be one where the organs act as input mechanisms to the lateral line nerve. The constant maintenance of these complex relations probably relies on genetic mechanisms and requires a certain amount of energy to create them and keep them constant.

Biologists now think the lateral line system helps to keep track of things in the environment of the fish such as water current and movement, but for some time, nothing was known about the function of this system. Still, though no function was known, the above mentioned complex spatial relations and constancy in conspecifics provided reason to think that the lateral line system did serve some fitness enhancing function (Nichols \& Grantham, 2000, p. 657). According to Nichols and Grantham, biologists did in fact consider the system to be an adaptation before they had a clear idea of its function.

Even though they do not spend much time developing a detailed account of complexity, Nichols and Grantham seem to adopt the view that an organ or structure is complex if it is composed of many interacting parts. Their reasons for holding that such complexity must be accounted for by natural selection is that 1) it is unlikely that undirected and unselected variation could produce and coordinate the actions of these parts and 2) the complex trait/organ would deteriorate unless favored by selection (Nichols \& Grantham, 2000, pp. 655 \& 657). Nichols and Grantham set out to argue that phenomenal consciousness is analogously complex to structures like the lateral line system which require adaptive explanations. To do so they cite two aspects of phenomenal consciousness: the unity of consciousness and dissociations from neuropsychology. A dissociation can be thought of as showing two things to be of a different sort. Particularly, these involve showing that two cognitive phenomena are separate. For example, blindsight points to a dissociation between the phenomenon of visual awareness and of taking in visual information. By citing various dissociations, such as blindsight, Nichols and Grantham seek to establish a picture of phenomenal consciousness as a system, or central mechanism, that receives inputs from various sensory modalities. I will focus on Nichols and Grantham's use of dissociations and not their use of the unity of consciousness. I accept that our experience seems unified to us and that whether there is one system of p-consciousness or a collection of connected systems that give this effect is irrelevant to their argument.

In addition to blindsight, some of the dissociations that Nichols and Grantham cite include prosopagnosia and achromatopsia. Prosopagnosic patients are not able to overtly recognize familiar faces but are able to show covert recognition of familiar faces after brain damage. When shown familiar faces and read a list of names, for example, patients' behavioral and physiological reactions show covert recognition. However, when asked which name belongs to the face, patients test at chance levels (Young, 1994, 179-182). Achromatopsic patients show some signs of sensitivity to colors in visual processing, yet lack any awareness of color. Similarly, Nichols and Grantham cite dissociations from other sensory modalities involving auditory and tactile stimuli.

Since there seem to be many cases where subjects show an ability to take in certain amounts of perceptual information but lack phenomenal consciousness of that information, Nichols and Grantham suggest that the correct explanation of the situation is that there are many inputs, which are outputs of functionally described sensory modules and which in these cases are damaged, that feed into a phenomenal consciousness system. The variety of inputs which feed into the phenomenal consciousness system are then taken as evidence that phenomenal consciousness is complex in the same way that the lateral line system is complex.

Both systems have several independent input channels that feed into a more central mechanism. Just as it takes energy to develop and maintain the network of channels and lateral line organs in fish, it must take energy to create and maintain a system of multiple channels that provides input into phenomenal consciousness. If this kind of structure did not perform any function, it would passively deteriorate or be actively selected against (Nichols \& Grantham, 2000, p. 663). Since the sort of evidence which they invoke to establish the existence of 
distinct input channels into the p-consciousness system is functional in nature, Nichols and Grantham assert that they must be realized by some type of neural structure. And of course, that kind of complexity, they argue, must serve a function of some sort to be maintained and warrants classification as an adaptation.

In summary then, Nichols and Grantham's argument comes in two parts. First, a complex structure is one comprised of many interacting parts. It is unlikely that undirected and unselected variation could produce and coordinate the actions of these parts. The complex trait/organ would deteriorate unless favored by selection. Thus, a complex structure is most likely a product of natural selection, i.e. is an adaptation.

The second part of the argument runs as follows. Dissociations from cognitive neuropsychology show that patients can be sensitive to perceptual information without phenomenal consciousness (awareness) of that information while still aware of other perceptual information from other senses. The best explanation of this situation is that phenomenal consciousness is a system, or mechanism, which receives input from different input channels. Phenomenal consciousness must be realized physically in the neural-stuff in the brain. Thus, phenomenal consciousness is a complex structure. Thus, phenomenal consciousness is most likely a product of natural selection, i.e. is an adaptation.

\section{FUNCTIONAL CHARACTERIZATIONS}

In this section, I would like to raise a few questions that I think challenge the effectiveness of Nichols and Grantham's argument in establishing that there is a system dedicated to phenomenal consciousness. In particular, I would like to challenge their premise that a neural structure dedicated solely to the functional work they have set out for it is established by the neuropsychological evidence. However, even if this premise is not necessary for their argument, i.e. a purely functional and complex description is enough to warrant the conclusion that such a functional trait is an adaptation, the argument still fails to establish its conclusion. The argument fails because there seem to be various alternative explanations of some of the dissociations employed by Nichols and Grantham. The existence of plausible, alternative accounts does not allow them to make the inference that the best explanation of the dissociations is one that employs a pconsciousness system.

Cognitive psychologists, neuroscientists and neuropsychologists typically search for and require double dissociations before they can make a case for the existence of a functionally encapsulated system. However, Nichols and Grantham only provide single dissociations. In a related vein, their interpretation seems to employ a view of the mind which is modular in nature and which also suggests an assumption of locality. Though there may be some cognitive activities that are aptly characterized by cognitive psychology and cognitive neuroscience as modular, there are others that are not.

I will touch on each of these criticisms below by beginning with Nichols and Grantham's functional characterization generally, then turn to possible alternative explanations of the dissociations cited and end with a few remarks on the issue of modularity. This final section will include the criticism that Nichols and Grantham do not employ rigorous enough criteria by not providing, at the least, double dissociations of the phenomena.

Functional Account and Implementational Account? Let us consider some of the presently relevant information. 1) People can have information (though not access consciousness if Block is right, and Nichols and Grantham accept his distinction) without awareness of information. This is based on the neuropsychological dissociations. 2) Without damage to brain areas, experiences seem rather unified. 3) Selective damage causes selective deficits. 4) Cognitive neuroscience, psychology and cognitive science generally posit systems, or construct functional descriptions, to explain phenomena and cognitive processes. Additionally, there is the picture we can keep in mind of Nichols and Grantham's explanation of the neuropsychological dissociations, i.e. the view of a p-consciousness system elaborately linked by inputs to each sensory modality and having some form of output to other functionally described task-masters.

First of all, in assessing these points it should be kept in mind that posited systems and functional descriptions do not always pan out. Furthermore, not only is the history of psychology littered with different explanatory programs, but there are numerous competing models on display at any given time. This is not very surprising since they are typically working hypotheses that represent rough sketches of how the mind might accomplish certain computational tasks, much like a flow chart. The map of systems and their interactions can become more accepted as fairly accurate cognitive maps, but the evidence that Nichols and Grantham offer (only dissociations from brain damaged subjects) is probably not sufficient on its own to establish the view of phenomenal consciousness as the type of system that they are pushing for.

For one thing, it begs empirical questions regarding how best to interpret cognitive deficits based on damage to the brain. Does damage to a particular area only affect information processing in that area of the brain, or does it have a wider influence on other structures and processes? Does the lesion/damage in question completely eliminate processing through that channel, 
or does it merely degrade its quality? If, for example, the quality of the signal is the important factor in whether or not one becomes p-conscious of some information, it is not necessary to suppose that there is a separate system that is not receiving a signal. Instead, the explanation may be that the signal is not sufficiently strong or of a certain sort. So there might be alternative computational accounts of the dissociations which do not employ a p-consciousness system. ${ }^{2}$

The level of abstractions that the cognitive maps represent, in particular Nichols and Grantham's, are not typically or strongly constrained by the actual hardware that implements them, which in this case is the brain. This undermines, in some respects, their attempt to argue from the picture of phenomenal consciousness as a system to the supposed fact that it is an anatomical structure which mirrors the connections they purport it to have and which the lateral line system has. This is, of course, what they must be able to show if they are going to show that it is analogous to the lateral line system and to show that it is a product of natural selection. To fill out the view of phenomenal consciousness as a system in itself, more information is required, such as what type of representations and computations are being employed in this system and how this system is implemented in the brain (cf. Marr 1982).

The problem is that even if the functional characterization of the various inputs, connections and systems are fairly accurate functional characterizations, they may be realized in very different ways in the brain. Even granting that there is some "place" where pconsciousness occurs, it could easily be the case that it is also the "place" where a-consciousness or some other activity occurs. This would allow for phenomenal consciousness to be a free-rider with respect to its selective history. Without recourse to a specific functional role for $\mathrm{p}$-consciousness, which Nichols and Grantham admit not having, they cannot assume that their functional (or quasi-functional) schema is sufficient to establish an anatomically complex system for $\mathrm{p}$-consciousness.

To sum up, Nichols and Grantham want to show phenomenal consciousness is anatomically complex. But the characterization they provide is quasifunctional, and it must map onto unique brain/neural structures if it is to be anatomically complex and require an adaptive explanation. But there is no reason to think that a quasi-functional characterization will map onto unique neural structures. The brain structure that they assume subserves, and is dedicated to, $\mathrm{p}$-consciousness ${ }^{3}$ might just as easily subserve some other functions, and so, not provide an anatomically complex system for phenomenal consciousness.

\section{Alternative Explanations}

We still do not have enough reason to suppose that there is a complex anatomical structure for phenomenal consciousness. Suppose though, as may well be the case, that there is no reason why the p-consciousness system needs to be realized in a particular anatomical structure in order to qualify as a complex structure. That is, suppose that its functional complexity, as mapped out by the picture of it as receiving inputs from the sensory modalities and as having its own outputs to other functional areas, is sufficient to establish its complexity as a functional entity. After all, biologists talk about complex, adaptive behaviors where the behavior is functionally described. Though this does not seem to fully capture the relevant force of their argument and the analogy with the lateral line system in fish, we can grant them this interpretation and still show that their argument is not sufficient to establish its conclusion. The problem, again, is that there are a variety of other options available that can explain the same neuropsychological data. That is, we do not have to accept Nichols and Grantham's functional picture that they think best explains the dissociations.

Farah (1997) distinguishes between three different general sorts of neuropsychological accounts given to explain the relations between consciousness and brain states. These general types of accounts provide a different functional characterization and analysis of conscious states. The types generally are: 1) Consciousness as the privileged role of particular brain systems, 2) Consciousness as a state of integration among distinct brain systems and 3) Consciousness as a graded property of neural information processing. Nichols and Grantham fit into the first category. The second category holds that there is no system dedicated to consciousness but that consciousness arises through the dynamic interaction of various systems and their representations. The idea is that they become consistent or share certain characteristics which then bind them together. For example, Crick and Koch's (1990) well known hypothesis regarding neural oscillation rates is one example of this type of account. The third category is similar to the suggestion presented above, namely, that the quality of the neural signal is correlated with awareness of the information represented.

Farah (1997) examines how these three different accounts fare in their explanations of blindsight, prosopagnosia, perception in neglect and extinction, and implicit reading in pure alexia. Interestingly, each of the syndromes requires an explanation unique to its special circumstances.

In blindsight, there are two possible neural pathways involved, with each supporting a different general account (type 1 and type 3). Prosopagnosia and perception in neglect and extinction both seem equally explained by account types 2 and 3. Implicit reading seems to be better explained by account type 1. A possible implication of this is that consciousness might not be the sort of thing that is subserved by, or can be 
explained by reference to, one account. This would seem unlikely if consciousness were a complex but unified system.

In addition, Farah asserts that these syndromes are not as clean and precise as they are often imagined to be. Cases such as blindsight are not simple cases of normal perception with no awareness. Rather, the level of perception involved is nowhere near normal, and thus the dissociations should not be taken to show a complete distinction between perception and awareness of perception (Farah, 1997, pp. 232-233). Farah takes this to imply that there " ....is currently no evidence for a dedicated consciousness awareness system, distinct from the systems that perform specific perceptual or cognitive functions" (Farah, 1997, pp. 233).

The above considerations imply that Nichols and Grantham's interpretation of the neuropsychological dissociations is not the only one available and that the dissociations are not alone sufficient to establish that there is a p-consciousness system. Furthermore, even if their account is the best explanation for, say, blindsight, there is reason to question whether it can adequately explain the other dissociations. Moreover, it might be unlikely that only one account will suffice to explain all of the dissociations since they are behaviorally and physiologically very different.

\section{Modularity}

The above remarks are related to a more general issue I would like to comment on regarding the adequacy of a modular view of the mind. It seems that Nichols and Grantham end up assuming that a modular view of the mind generally, and phenomenal consciousness in particular, is the correct way to envision the mind. ${ }^{4}$

A module in cognitive science is roughly seen as an informationally encapsulated mechanism that is dedicated to receiving some input, performing some operation or function on the input and then propagating an appropriate output. On a strong modular view, the modules, or boxes, do not generally interact except when one sends its output to the next module. Each module also generally has its own form of representations and computations that it employs.

It seems plausible that there are some cognitive activities, for example initial sensory processes, that are best viewed of as modular. For instance, the MullerLyer illusion, where two lines with arrows on the end are actually equal in length, appears to the observer to be a case where one line persistently looks longer even though the observer knows that they are equal in length (Appelbaum, 1998, p. 627). This is a possible example of modularity because the knowledge that the lines are equal is unable to penetrate and influence the perception that one line is longer than the other.

However, there are good reasons to think that some cognitive processes must be nonmodular. For example, abductive inferences are probably more global processes (Fodor 2000). In fact, any process, including inferences, which must make use of multiple sources of information are likely to be nonmodular. Modular accounts also have difficulty explaining how ideas can arise that are independent of perceptual information and that involve mixed contents, i.e. contents that involve a variety of domains or are not specifically domainspecific. ${ }^{5}$

Additionally, gradual degradation of cognitive abilities with selective damage, as opposed to complete obliteration, seems to suggest that strong modularity might be, well, too strong. If modularity is accompanied with the assumption of locality, ${ }^{6}$ then damage to a particular component of a system will only affect that component, leaving the other components to function as they did before the damage. Cognitive neuropsychology employs this assumption regularly in inferences from dissociations to characterizations of functional architectures. Indeed, this assumption seems at play in the reasoning displayed by Nichols and Grantham in their construction of the phenomenal consciousness system and its many inputs from the selective damage at play in the dissociations.

However, the necessity of the locality assumption is called into question by Farah (1994) who designed three separate computational models that were based on neuropsychological data of dissociations involving knowledge of living things, disengagement of visual attention and face recognition. The models did not incorporate the locality assumption, but instead utilized assumptions that information processing is distributed, interactive and graded. The dissociation data along with these assumptions allowed Farah to construct plausible models of functional architecture utilizing connectionist neural networks which, though damaged, were able to account for the same results seen in the cognitive deficits of real patients (Farah 1994). ${ }^{7}$ She argues that these models are more plausible than modular accounts based on locality because they accord with other data regarding brain organization and provide simpler accounts of cognitive processing (Farah 1994).

In addition to the above concerns over modularity, neuropsychological dissociations are not typically enough to establish modularity. Alternative explanations, again, abound. To try and make firmer the inference to modularity from a certain deficit due to brain damage, neuropsychologists try to establish double dissociations. A double dissociation is displayed when a different subject shows the opposite dissociation from the first. For example, in cases where patients with brain damage can read aloud accurately but without being able to grasp the semantics of the words (e.g. cannot classify words into their semantic categories or match a printed word to its pictorial equivalent), one cannot infer that there is a conceptual subsystem which 
deals primarily with semantic associations, along with a visual processing system and a vocal motor output system. The dissociation might just as well be explained by assuming that there is only one large system and that semantic classification is actually a more difficult task which is hindered by the weakened system. What is needed is a double dissociation. This would involve showing a case of the opposite situation where the patient has damage elsewhere and lacks the ability to either read aloud or write but maintains an ability for conceptual and semantic tasks.

Double dissociations are usually taken to imply modularity (Appelbaum, 1998, pp. 633). However, as Appelbaum points out, even double dissociations do not strictly imply modularity, they only fit nicely together. The implication might be stronger if there were only two options for cognitive systems: a general purpose system or modular systems, but there are a variety of possibilities that Appelbaum notes including overlapping processing regions, multilevel systems, coupled systems and semi-modules (Appelbaum, 1998, p. 633). In fact, some of these various systems were used by Farah in her models mentioned above.

Given the dubious nature of inferring the existence of modules based only on neuropsychological dissociations, it would seem that Nichols and Grantham should take more care to try and present cases of double dissociations to strengthen their conclusion. However, even if they were to present double dissociations, there would still be room for serious doubt as to whether they were sufficient to establish the intended conclusion that p-consciousness is a complex system in its own right which demands an evolutionary explanation.

There is also a further obstacle to conceiving of phenomenal consciousness as modular. If it were encapsulated, and since the modules require computations on the syntactic properties of representations, it would be imperative to make clear what type of representations are employed in the phenomenal consciousness module, what the syntax of such representations is, and what sorts of operations are performed on them. This, in itself, is not the problem since this problem faces any adequate account of mental processes in cognitive science. The problem is that it is not clear that phenomenal consciousness is representational. Block (1995) for example, holds that there are some instances of p-consciousness which are not representational (e.g. orgasms). If it turns out that $p$ consciousness is not representational, then other tools would be necessary to explain it.

\section{QUESTIONS OF CONSISTENCY}

A final objection to Nichols and Grantham that I would like to briefly state involves consistency. The reason for arguing that p-consciousness is a system is due to the acceptance of Block's distinction between aconsciousness and p-consciousness. Recall that accepting this distinction forced them to set aside the neuropsychological data as evidence of the function of consciousness. Given the distinction, the data can no longer provide evidence for the function of phenomenal consciousness because it is possible that the functional deficit is actually due to a lack of access consciousness. However, once Nichols and Grantham accept the distinction, they proceed to put it aside and go along with their cases of dissociations to show that there is a system of phenomenal consciousness.

The problem is that once they acknowledge the distinction and relation between the two, they cannot, without argument, suppose that these same dissociations show there is a system of p-consciousness and ignore that they might just as well be pointing toward a system of a-consciousness. If the dissociations conflate the two aspects of consciousness in regard to the function of $p$ consciousness, they must also conflate the two aspects of consciousness in regard to the system, or systems, that the dissociations allegedly demonstrate. In other words, they acknowledge the distinction between a- and p-consciousness, but they ignore the distinction in regards to establishing the $\mathrm{p}$-consciousness system. But if the distinction is real, it should apply in both cases. Thus, their demonstration of a phenomenal consciousness system must take into consideration the distinction. But it does not, and so they have not demonstrated that a system for $\mathrm{p}$-consciousness exists. Nichols and Grantham apparently think that the burden of proof shifts to someone who thinks a-consciousness might be at play in the phenomena related to the dissociations, but it seems that once they acknowledge the problem that there could be a conflation in systems underlying the dissociations, they should do more to solve it than sweep it under the rug. However, ignoring this possibility in the complex system argument makes the conclusion that there is a p-consciousness system that is an adaptation less reliable.

\section{WHERE TO GO FROM HERE: CHALLENGING THE DISTINCTION}

All of this so far, has been somewhat negative. I think that Nichols and Grantham's argument from complexity fails because they fail to establish that phenomenal consciousness is a complex structure in the relevant sense. However, I would like to have something positive to say. Given the resources of cognitive science and its allegiance to information processing, it is at least conceivable that the information processing aspects of consciousness stand a chance of being illuminated. However, the phenomenal aspect of consciousness and its prospects for an adequate explanation (whatever that might be) are much fuzzier. There are attempts to formulate representational accounts of phenomenal 
consciousness (Dretske 1995; Tye 1995) which might make the chances of an explanation more feasible. Or it is possible that cognitive science can develop new explanatory constructs that go further towards explaining a non-representational notion of phenomenal consciousness. However, these are problems for explaining more aspects of consciousness than Nichols and Grantham are interested in explaining. Their concern in explaining the evolutionary function and causal role function of consciousness does not seem to require a complete explanation of consciousness.

I think that their cause, though, might be better served by adopting a different strategy. Some of the difficulties in understanding the roles of consciousness begin by acknowledging Block's distinction. If there is no distinction, then the differences in behavioral abilities of patients with blindsight, etc. can be compared to normal subjects to provide evidence of a function of consciousness. Though there may be some examples of abilities that are still possible without phenomenal consciousness, they are greatly hindered.

Are there reasons to refuse Block's distinction? One might be inclined to ask what the grounds for the distinction are. Block (1995) admits throughout his paper that it is a conceptual distinction and that it might not actually occur. He does offer some examples that he suggests show the possibility of having p-consciousness without a-consciousness and vice versa, but they are open to interpretations that negate his. Block's examples of a-consciousness without p-consciousness include a zombie, which he admits is very controversial, and the possibility of superblindsight which he himself admits there are no actual cases of (Block 1995, 233).

His example of p-consciousness without aconsciousness involves a version of the following story. Suppose you are in your office talking to a student when at noon you suddenly realize that outside of your window there is an amazingly noisy drill in operation. You also realize that the drill has been operating for quite some time. Block insists that you were pconscious of the noise all along but only became consciously aware, that is a-conscious and p-conscious, of the noise at noon (Block 1995, 234). Thus, the two are distinct. What are we to make of such a claim?

First, it is not clear at all that you were ever actually aware of the noise before noon. You might have just become aware of it but inferred that it had probably been operating for a while. This might lead you to say that you were aware, in a loose way, that it had been operating before noon without actually being aware of its operation before noon. Block's insistence that you were aware of it before noon seems to be merely a reliance on what one might commonly say in such a situation. However, common linguistic uses should not be taken to reveal metaphysical truths such as that you actually were in a perceptual state, experiencing the drill's noise, but that you just didn't realize it until later. Maybe that is true. Maybe it is not. But if Block wants to convince us that it is, he had better provide more of an argument.

Suppose, however, that Block is right. You were aware of the noise before noon. As Jennifer Church (1995) points out, the options available to you are quite extensive given that a-consciousness involves accessibility. Since you do eventually access the noise, the experience of hearing it has access potential and the experience contains accessibility.

There also seems to be a sense in which one thinks that the drill's noise would have been accessed sooner if it had been more important; if, for example, it had been the sound of a bomber zeroing in or if the drill had begun drilling through the wall. yFurthermore, maybe the noise was accessed, contra Block, and did play a role in initiating some form of behavior such as raising your voice or moving closer to whom you were speaking with (Church, 1995, p. 252). All of these seem as likely as, if not more so than, having been aware of it even though you did not know it or access the noise. And if any of these possibilities were to obtain in the scenario, then it would not be a case of p-consciousness without a-consciousness.

Since reflections such as those above are the sort that motivate the distinction between a-consciousness and pconsciousness, it seems prudent to refuse, or at least delay, allowing the distinction. Furthermore, there are questions about what a-consciousness could be consciousness of if it did not have phenomenal characteristics. If it is a state that contributes to consciousness, then it is just a piece of the puzzle and not properly consciousness. If it is an object of consciousness, then it cannot be separate from phenomenal consciousness since it will be what you are conscious of and there will be something it is like to be conscious of it.

Owen Flanagan's (1997) approach seems more reasonable. He also sees no reason in accepting Block's distinction but thinks that there is plausibility in accepting a distinction between “...informational sensitivity, which involves access to information without phenomenal awareness, and experiential sensitivity, which involves both phenomenal feel and access to information..." (Flanagan, 1997, p. 369). This type of distinction is actually what is warranted by many of the dissociations already discussed. In blindsight, the subject lacks phenomenal awareness of what is in their impaired visual field, yet is to some 
degree still sensitive to the information in that visual field. In cases where a subject does have phenomenal awareness of some information, they also have access to that information, at least in the sense that they have access to what state they are in.

On Block's distinction one is forced into saying that though the blindsight subject does have access to some of the information in their damaged visual field, they are nonetheless not a-conscious of it. But since the blindsight patients can use this information, albeit with less force, to affect their behavior and responses to certain tasks, it seems that Block's distinction is arbitrary.

If this distinction is denied, then careful considerations of neuropsychological dissociations may be able to play a stronger role in making the case for a function of consciousness. The tasks that cannot be adequately performed in many of the scenarios could plausibly be seen as deficits explained by the lack of consciousness of the relevant phenomena. The above reasoning would not be subject to the same type of criticisms aimed at Nichols and Grantham since there would be no reliance or appeal to complexity, structures, or modules. More importantly, there is no mention of an evolutionary explanation for the origin and function of consciousness.

\section{CONCLUSION}

Evolutionary accounts can either develop adaptive explanations for an acknowledged trait, or they can proceed by speculating on likely encountered survival problems and develop stories about optimal solutions to those problems, positing mechanisms which have as their function, performing these solutions. Davies (1996) has argued that evolutionary speculations about the history of a particular trait, or mechanism, are generally fruitful only if that trait is fairly well understood. Attempts to use evolutionary considerations to predict, discover or understand psychological phenomena are mistaken because that task is already in the domain of cognitive science and is prior to the elaboration of evolutionary accounts of those phenomena (Davies 1999; Grantham and Nichols 1999). In the case of psychological mechanisms, historical investigations can only be fruitful once the cognitive architecture is sufficiently understood. In the case of phenomenal consciousness, however, the cognitive architecture is not well understood. There are competing explanations and insufficient amounts of evidence to establish one over the other. This problem presents a large obstacle to Nichols and Grantham's (2000) project.

Furthermore, Nichols and Grantham's argument that phenomenal consciousness is an adaptation fails because they fail to establish that it is a complex anatomical structure. This is partially due to over- estimations of interpretations of neuropsychological data regarding dissociations generally, and their own in particular. Single dissociations alone are not sufficient to establish the existence of a system or module dedicated to some function. Minimally, double dissociations are required. However, even if they were to establish a double dissociation, they would have to counter rival explanations of the same phenomena. Furthermore, it may be overzealous to expect one functional characterization to be able to account for a variety of different cognitive deficits. It may be a more fruitful strategy to deny Block's distinction between aconsciousness and p-consciousness, allowing for some of the neuropsychological data to provide evidence for the causal-role function of consciousness.

Though it was not discussed in this paper, there are further questions regarding both Nichols and Grantham's argument and general question of interest. For example, what is the status of their claims that it is unlikely that undirected and unselected variation could produce and coordinate actions of interconnected parts? Are there examples of nonadaptations which might be complex? Would complex traits or organs deteriorate unless favored by selection? If so, how do we make sense of entities that were selected for a past function but which no longer fulfill that function? Does this ignore the distinction between historical and current uses? More generally, what is the motivation behind, and value of, trying to give evolutionary explanations of psychological traits or traits where the history of that trait is cloudy at best? These seem to me to be interesting questions and ones which require a more detailed treatment than I can give here.

\footnotetext{
Notes

${ }^{1}$ At the very least it blurs the distinction between creature consciousness and state consciousness.

${ }^{2}$ In fact there are both competing accounts of functional descriptions and of how to interpret them, as well as some disagreement on what neural pathways are involved in certain of the dissociations, e.g. blindsight. See Farrah 1997.

${ }^{3}$ It is important to note that there is no known or agreed upon neuroanatomical localization of consciousness.

${ }^{4}$ Some advocates of modularity apparently draw distinctions between modules and central systems (Appelbaum 1998). The idea is that central systems are not modules because they are occupied with inferences and/or belief fixation whereas modules are viewed as relay stations for information (often perceptual) to central systems (Appelbaum 1998). Even if this characterization is taken at face value, the type of system which Nichols and Grantham make p-consciousness out to be seems understandable as a module since it is not active in making inferences but is viewed as an integration of
} 
perceptual information which is relayed to other control mechanisms (Nichols and Grantham 2000, 667-668).

${ }^{5}$ Though, see Carruthers (2003) for an attempt at squaring modular accounts with these problems.

${ }^{6}$ The locality assumption is the assumption that damage to one module or part of the functional architecture of a system will only affect that component such that all other components will continue to function normally.

${ }^{7}$ Connectionist networks in general are excellent examples of models of cognitive processes that do not, or need not, rely on modularity. Though there is controversy over how well they represent activity in the actual brain, there is common reliance on them to model and test various cognitive hypotheses. To the extent that they are applicable to human cognition, they provide some reason to think that the human mind is not predominantly modular.

\section{REFERENCES}

Appelbaum, I. (1998). Modularity. In W. Bechtel \& G. Graham (Eds.), A Companion to cognitive science. Malden, MA: Blackwell Publishers.

Block, N. (1995). On a confusion about a function of consciousness. Behavioral and Brain Sciences, 18, 227-287.

Barkow, J., Cosmides, L., and Tooby, J. (Eds.). (1992). The adapted mind: evolutionary psychology and the generation of culture. New York: Oxford University Press.

Carruthers, P. (2003). On fodor's problem. Mind and Language, 18, 502-523.

Churchland, P.S., \& Sejnowski, T.J. (1992). The computational brain. Cambridge, MA: MIT Press.

Crick, F., \& Koch, C. (1990). Function of the thalamic reticular complex: The searchlight hypothesis. Seminars in the Neurosciences, 2, 287-296.

Davies, P.S. (1996). Discovering the functional mesh: On the methods of evolutionary psychology. Minds and Machines, 6 , 559-585.

Davies, P.S. (1999). The conflict of evolutionary psychology. In V. Hardcastle (Ed.), Where biology meets psychology, 6781. Cambridge, MA: MIT Press.

Dretske, F. (1995). Naturalizing the mind. Cambridge, MA: MIT Press.

Farah, M.J. (1994). Neuropsychological inference with an interactive brain: A critique of the "locality" assumption. Behavioral and Brain Sciences, 17, 43-104.

Farah, M.J. (1997). Visual perception and visual awareness after brain damage. In N. Block, O. Flanagan, \& G. Guzeldere (Eds.), The nature of consciousness: philosophical debates, 203-236. Cambridge, MA: MIT Press.

Flanagan, O. (1997). Conscious inessentialism and the epiphenomenalist suspicion. In N. Block, O. Flanagan, and G. Guzeldere (Eds.), The nature of consciousness: philosophical debates, 357-373. Cambridge, MA: MIT Press.

Fodor, J.A. (2000). The mind doesn't work that way. Cambridge, MA: MIT Press.

Godfrey-Smith, P. (1996). Complexity and the function of mind in nature. New York: Cambridge University Press.

Grantham, T. \& Nichols, S. (1999). Evolutionary psychology: ultimate explanations and panglossian predictions. In V.
Hardcastle (Ed.), Where biology meets psychology, 47-66. Cambridge, MA: MIT Press.

Hardcastle, V. (Ed.). (1999). Where biology meets psychology: Connections, constraints, conjectures. Cambridge, MA: MIT Press.

Hardcastle, V. (1996). How to build a theory in cognitive science. Albany: State University of New York Press.

McNamara, T.P. (1999). Single-code versus multiple-code theories in cognition. In R.J. Sternberg (Ed.), The nature of cognition. Cambridge, MA: MIT Press.

Marr, D. (1982). Vision: A computational investigation into the human representation and processing of visual information. New York: W.H. Freeman and Co.

Nagel, T. (1974). What is it like to be a Bat? Philosophical Review, 83, 435-450.

Nichols, S. \& Grantham, T. (2000). Adaptive complexity and phenomenal Consciousness. Philosophy of Science, 67, 648670 .

Okasha, S. (2003). Fodor on cognition, modularity, and adaptationism. Philosophy of Science, 70, 68-88.

Polger, T. \& Flanagan, O. (1999). Natural answers to natural questions. In V. Hardcastle (Ed.), Where biology meets psychology, 221-247. Cambridge, MA: MIT Press.

Revonsuo, A. \& Kamppinen, M. (Eds.). (1994). Consciousness in philosophy and cognitive neuroscience, 173-203. Hillsdale, NJ: LEA.

Shapiro, L.A. (1999). Presence of mind. In V. Hardcastle (Ed.), Where biology meets psychology, 83-98. Cambridge, MA: MIT Press.

Tye, M. (1995). Blindsight, orgasm, and representational overlap. Behavioral and Brain Sciences, 18, 268-269.

Young, A.W. (1994). Neuropsychology of awareness. In A. Revonsuo and M. Kamppinen (Eds.), Consciousness in philosophy and cognitive neuroscience, 173-203. Hillsdale, NJ: LEA. 